\title{
Magnetic Resonance Imaging of the Head and Neck with and without Contrast
}

National Cancer Institute

\section{Source}

National Cancer Institute. Magnetic Resonance Imaging of the Head and Neck with and

without Contrast. NCl Thesaurus. Code C137918.

Magnetic resonance imaging of the head and neck with and without the use of a

contrast agent to enhance the image. 